\title{
Lopinavir-ritonavir versus hydroxychloroquine for viral clearance and clinical improvement in patients with mild to moderate coronavirus disease 2019
}

Ji-Won Kim ${ }^{1}$, Eun Jin $\mathrm{Kim}^{2}$, Hyun Hee Kwon³ , Chi Young Jung ${ }^{2}$, Kyung Chan Kim², Jung-Yoon Choe ${ }^{1}$, and Hyo-Lim Hong ${ }^{3}$

Divisions of ${ }^{1}$ Rheumatology, ${ }^{2}$ Pulmonology, and ${ }^{3}$ Infectious Diseases, Department of Internal Medicine, Daegu Catholic University School of Medicine, Daegu, Korea

Received: May 13, 2020 Revised : May 27, 2020 Accepted: May 27, 2020

\section{Correspondence to} Hyo-Lim Hong, M.D. Department of Internal Medicine, Daegu Catholic University Medical Center, 33 Duryugongwon-ro 17-gil, Nam-gu, Daegu 42472, Korea Tel: +82-53-650-4899 Fax: +82-53-623-7507 E-mail:hlhong@cu.ac.kr https://orcid.org/0000-00032147-1381
Background/Aims: The efficacies of lopinavir-ritonavir or hydroxychloroquine remain to be determined in patients with coronavirus disease 2019 (COVID-19). To compare the virological and clinical responses to lopinavir-ritonavir and hydroxychloroquine treatment in COVID-19 patients.

Methods: This retrospective cohort study included patients with COVID-19 treated with lopinavir-ritonavir or hydroxychloroquine at a single center in Korea from February 17 to March 31, 2020. Patients treated with lopinavir-ritonavir and hydroxychloroquine concurrently and those treated with lopinavir-ritonavir or hydroxychloroquine for less than 7 days were excluded. Time to negative conversion of viral RNA, time to clinical improvement, and safety outcomes were assessed after 6 weeks of follow-up.

Results: Of 65 patients (mean age, 64.3 years; 25 men [38.5\%]), 31 were treated with lopinavir-ritonavir and 34 were treated with hydroxychloroquine. The median duration of symptoms before treatment was 7 days and 26 patients (40\%) required oxygen support at baseline. Patients treated with lopinavir-ritonavir had a significantly shorter time to negative conversion of viral RNA than those treated with hydroxychloroquine (median, 21 days vs. 28 days). Treatment with lopinavir-ritonavir (adjusted hazard ratio [aHR], 2.28; 95\% confidence interval [CI], 1.24 to 4.21 ) and younger age (aHR, 2.64; 95\% CI 1.43 to 4.87 ) was associated with negative conversion of viral RNA. There was no significant difference in time to clinical improvement between lopinavir-ritonavir- and hydroxychloroquine-treated patients (median, 18 days vs. 21 days). Lymphopenia and hyperbilirubinemia were more frequent in lopinavir-ritonavir-treated patients compared with hydroxychloroquine-treated patients.

Conclusions: Lopinavir-ritonavir was associated with more rapid viral clearance than hydroxychloroquine in mild to moderate COVID-19, despite comparable clinical responses. These findings should be confirmed in randomized, controlled trials.

Keywords: COVID-19; Severe acute respiratory syndrome coronavirus 2; Lopinavir-ritonavir; Hydroxychloroquine 


\section{INTRODUCTION}

Since a novel coronavirus disease 2019 (COVID-19) emerged in Wuhan, China in December, 2019, it has spread worldwide resulting in the COVID-19 pandemic. The causative virus of COVID-19 was identified and named severe acute respiratory syndrome coronavirus 2 (SARS-CoV-2). SARS-CoV-2 causes upper and lower respiratory tract infections in humans and the clinical course can be fatal if the illness progresses to an acute respiratory distress syndrome (ARDS) [1-3]. More than 3 million cases with more than 200,000 deaths had been confirmed worldwide as of late April, 2020 [4]. However, effective antiviral agents targeting SARS-CoV-2 have not yet been developed. Lopinavir-ritonavir and chloroquine are each considered to have potential therapeutic benefits in SARS-CoV-2 infection based on previous preclinical and clinical data from SARS-CoV-1 and Middle East respiratory syndrome coronavirus (MERS-CoV).

Lopinavir-ritonavir was approved for treatment of human immunodeficiency virus (HIV) infection in 2000. Lopinavir is a HIV-1 protease inhibitor and a combination with another protease inhibitor, ritonavir, increases serum concentrations of lopinavir through cytochrome P45o inhibition. In vitro assays found that lopinavir had inhibitory activity against SARS-CoV-1 [5,6] and MERS$\mathrm{CoV}$ [7]. Patients with SARS-CoV-1 infections who were treated with lopinavir-ritonavir exhibited favorable clinical outcomes compared with historical controls who did not receive lopinavir-ritonavir [5]. Case reports or series have suggested a possible therapeutic effect of lopinavir-ritonavir against SARS-CoV-2 [8,9]. A randomized, controlled, open-label trial conducted in Chinese patients with severe COVID-19 found no benefit of lopinavir-ritonavir with respect to time to clinical improvement compared with standard care [10]. However, that clinical trial included COVID-19 patients with relatively severe respiratory illness. Data on the efficacy of lopinavir-ritonavir in patients with mild to moderate COVID-19 are needed.

Chloroquine, initially developed as an antimalarial agent, has antiviral activity against a broad range of viruses including HIV and coronaviruses [11]. Due to its inhibitory effect on viral replication and immune activation, chloroquine has gained interest as a weapon against coronavirus outbreaks associated with severe re- spiratory illness. As in SARS-CoV-1 [12-14], chloroquine was found to potently inhibit SARS-CoV-2 infection in in vitro assays [15]. Hydroxychloroquine has similar therapeutic activity but less toxicity than chloroquine, properties that have enabled its long-term usage in autoimmune diseases. Yao et al. [16] found that hydroxychloroquine was a potent inhibitor of SARS-CoV-2 in vitro. A few clinical studies conducted in patients with COVID-19 support the usage of hydroxychloroquine, although the results need to be interpreted with caution due to the small sample size, non-randomized or non-controlled design, short follow-up period, and different dosing regimens used in these studies [17-20].

Clinical data regarding the efficacy of lopinavir-ritonavir or hydroxychloroquine on COVID-19 patients are currently lacking. Previous studies in COVID-19 patients investigated the efficacy of lopinavir-ritonavir or hydroxychloroquine compared with standard care. However, to date, there have been no published data comparing the efficacy of lopinavir-ritonavir and hydroxychloroquine in patients with COVID-19. Lopinavir-ritonavir and hydroxychloroquine have been available prior to the development of specific COVID-19 treatments. It is therefore important to determine which drug has superior viral and clinical effects to guide treatment choice during the COVID-19 pandemic. We compared the virological and clinical responses of COVID-19 patients treated with lopinavir-ritonavir or hydroxychloroquine and attempted to identify independent factors associated with negative conversion of SARS-CoV-2 RNA using real-world data with a follow-up period of 6 weeks.

\section{METHODS}

\section{Patients}

All hospitalized patients with COVID-19 who were treated with lopinavir-ritonavir or hydroxychloroquine at Daegu Catholic University Medical Center in South Korea from February 17 to March 31, 2020 were eligible for this retrospective cohort study. Infection was confirmed if SARS-CoV-2 viral RNA was detected (positive real-time reverse transcriptase-polymerase chain reaction [RT-PCR]) from nasopharyngeal and oropharyngeal swabs and/or sputum. Patients who were treated with lopinavir-ritonavir and hydroxychloroquine concur- 
rently and those who received lopinavir-ritonavir or hydroxychloroquine for less than 7 days were excluded. Lopinavir-ritonavir (400 and $100 \mathrm{mg}$, respectively) was administered twice daily and hydroxychloroquine 400 mg was administered once daily. The study protocols were approved by the Institutional Review Board of Daegu Catholic University Medical Center (CR-20-110) and informed consent was waived due to the retrospective study design.

\section{Outcome measures}

The primary outcome was time to negative conversion of viral RNA in nasopharyngeal and oropharyngeal swabs since the start of lopinavir-ritonavir or hydroxychloroquine therapy. Negative conversion was defined as two consecutive negative RT-PCR results at least 24 hours apart. The secondary outcome was time to clinical improvement (cessation of oxygen support or resolution of respiratory symptoms such as cough and/or sputum and normalization of body temperature below $37.5^{\circ} \mathrm{C}$ ) since the start of the therapy. The safety outcomes assessed were adverse events and serious adverse events occurring after the initiation of treatment. Adverse events were assessed and graded according to the Common Terminology Criteria for Adverse Events (version 5.0) published by the US National Cancer Institute of the National Institutes of Health.

\section{Assessment and monitoring of virological and clini- cal parameters}

The follow-up period for all patients was 6 weeks, except where the patients were discharged or transferred, or died prior to completion of follow-up. Serial nasopharyngeal and oropharyngeal swab specimens were obtained every 3 to 4 days after the start of therapy. If the PCR result was negative and the patient was in a stable clinical status, the next sample was obtained 24 to 48 hours later to determine whether the patient was a candidate for de-isolation. To assess the viral load of the swab specimens, RNA was extracted from the specimens and RT-PCR targeting the SARS-CoV-2 envelope (E) and RNA-dependent RNA polymerase (RdRp) genes was performed. The detection limit was a cycle threshold (Ct) value of 35. Serial PCR results were reported as positive, inconclusive, or negative: a positive result indicated both $\mathrm{E}$ and RdRp genes were detected; an inconclusive result indicated only one gene was detected; a negative result indicated neither gene was detected.

Symptoms such as cough, sputum, dyspnea, and diarrhea were assessed daily by two physicians (H.L.H. and C.Y.J.). Oxygen saturation and vital signs including body temperature were monitored daily. The degree of oxygen support at baseline and during therapy was assessed according to a seven-category ordinal scale as follows: (1) not hospitalized with resumption of normal activities; (2) not hospitalized, but unable to resume normal activities; (3) hospitalized, not requiring supplemental oxygen; (4) hospitalized, requiring supplemental oxygen; (5) hospitalized, requiring high-flow nasal oxygen therapy or noninvasive mechanical ventilation, or both; (6) hospitalized, requiring extracorporeal membrane oxygenation or invasive ventilation, or both; and (7) death [10]. Data regarding the aforementioned parameters, as well as concomitant antibiotic, glucocorticoid, or intravenous immunoglobulin (IVIG) administration, chest computed tomography (CT) scans, laboratory values, and coexisting conditions, were collected through medical chart review.

\section{Statistical analyses}

Numerical variables for the two groups were compared using an unpaired $t$ test or Mann-Whitney $U$ test, as appropriate. Comparison of categorical variables was conducted using chi-square test or Fisher's exact test. Kaplan-Meier curves were depicted and log-rank tests were performed to compare the virological and clinical responses between the lopinavir-ritonavir and hydroxychloroquine treatment groups. Transfer or death before negative conversion of viral RNA (or clinical improvement) was censored. Cox proportional hazards regression was performed to identify independent factors associated with negative conversion of viral RNA. Factors significant in the univariable analyses $(p<0.05)$ were included in a multivariable analysis. All statistical analyses were conducted using $\mathrm{R}$ language version 4.0.0 ( $\mathrm{R}$ Foundation for Statistical Computing, Vienna, Austria).

\section{RESULTS}

\section{Patients}

Among 123 COVID-19 patients hospitalized during the 


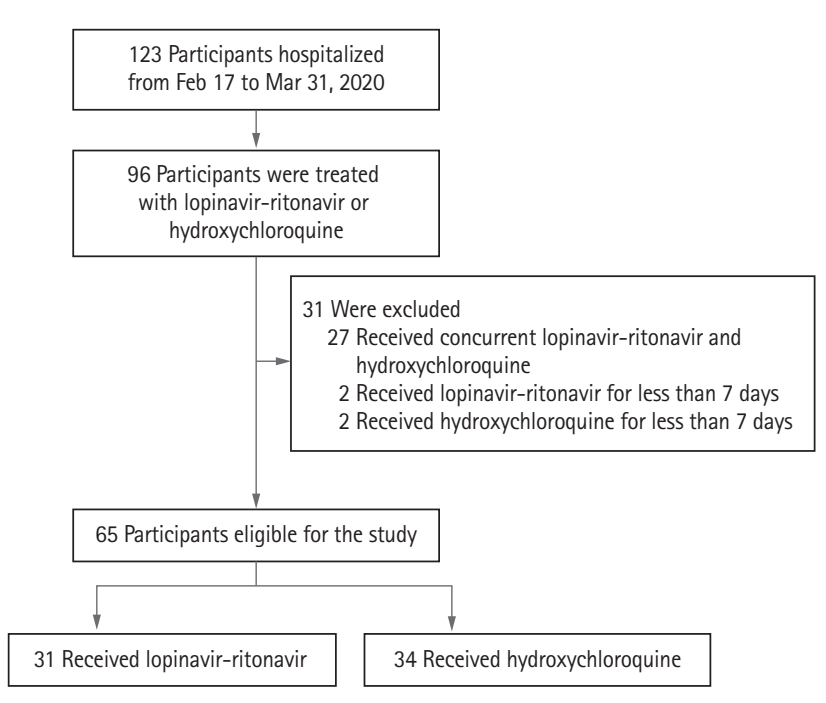

Figure 1. Flow chart of the study population.

study period, 96 patients were treated with lopinavir-ritonavir or hydroxychloroquine (Fig. 1). Excluding patients who received concurrent treatment with lopinavir-ritonavir and hydroxychloroquine $(\mathrm{n}=27)$, those who received lopinavir-ritonavir for less than 7 days $(n=2)$, and those who received hydroxychloroquine for less than 7 days $(n=2), 65$ patients were eligible for the study. Of these 65 patients, 31 received lopinavir-ritonavir over a median duration of 11 days (interquartile range [IQR], 10 to 14) and 34 received hydroxychloroquine over a median duration of 10 days (IQR, 10 to 13).

The characteristics of the patients at baseline are described in Table 1. The mean age was 64.3 years and 25 patients $(38.5 \%)$ were men. The median duration of symptoms before initiation of treatment with lopinavir-ritonavir or hydroxychloroquine was 7 days (IQR, 4 to 12). A total of 26 patients (40\%) required oxygen support at baseline, including two patients (3.1\%) who required high-flow oxygen. Age, sex, duration of symptoms before treatment, concomitant treatment with antibiotic, glucocorticoid, or IVIG, and coexisting conditions did not differ between patients who received lopinavir-ritonavir and those who received hydroxychloroquine. Patients treated with lopinavir-ritonavir tended to have more frequent bilateral multifocal ground-glass opacities (GGOs) and/or consolidations on their chest CT scans and required more common oxygen support at baseline and day 7 than those treated with hydroxychlo- roquine. Of the patients treated with hydroxychloroquine, nine patients $(26.5 \%)$ received concurrent azithromycin treatment.

\section{Virological response}

After 6 weeks of follow-up, 27 patients (87.1\%) treated with lopinavir-ritonavir and 21 patients $(61.8 \%)$ treated with hydroxychloroquine had negative conversion of viral RNA. Two patients were censored in the lopinavir-ritonavir group: one died and one was transferred to regional isolated living facilities for special care. Five patients were censored in the hydroxychloroquine group: one died and four were transferred to regional isolated living facilities for special care. Time to negative conversion of viral RNA was shorter in the lopinavir-ritonavir group than the hydroxychloroquine group (median, 21 days vs. 28 days; log-rank, $p=0.029$ ) (Fig. 2).

Next, we investigated factors associated with negative conversion of viral RNA in the total study population (Table 2). The results of the univariable analyses indicated younger patients ( $\leq 65$ years) and those who were treated with lopinavir-ritonavir were more likely to have negative conversion during the follow-up period $(p<0.05)$. Other variables, such as sex, duration of symptoms, oxygen support, concomitant therapy, chest CT findings, lymphopenia, elevated C-reactive protein (CRP), elevated aspartate aminotransferase (AST) or alanine aminotransferase (ALT), or the presence of diabetes, hypertension, cardiovascular disease, chronic lung disease, or chronic kidney disease, were not associated with negative conversion of viral RNA. In the multivariable analysis, use of lopinavir-ritonavir was an independent factor associated with negative conversion of viral RNA (adjusted hazard ratio [HR], 2.28; 95\% confidence interval [CI], 1.24 to 4.21 ) along with younger age (adjusted HR, 2.64; 95\% CI 1.43 to 4.87 ).

\section{Clinical response}

Two patients in the hydroxychloroquine group were excluded from the clinical response analyses: one showed clinical improvement before initiation of treatment and the other could not express symptoms due to cognitive dysfunction. After 6 weeks of follow-up, 30 patients (96.8\%) treated with lopinavir-ritonavir and 29 patients (90.6\%) treated with hydroxychloroquine showed clinical improvement. Time to clinical improvement was 
Table 1. Characteristics of the study population

\begin{tabular}{|c|c|c|c|c|}
\hline Characteristic & $\begin{array}{l}\text { Total } \\
(\mathrm{n}=65)\end{array}$ & $\begin{array}{l}\text { Lopinavir-ritonavir } \\
(\mathrm{n}=31)\end{array}$ & $\begin{array}{l}\text { Hydroxychloroquine } \\
(\mathrm{n}=34)\end{array}$ & $p$ value \\
\hline Age, yr & $64.3 \pm 15 \cdot 4$ & $64.3 \pm 14.6$ & $64.3 \pm 16.3$ & 1.000 \\
\hline Male sex & $25(38.5)$ & $11(35.5)$ & $14(41.2)$ & 0.829 \\
\hline $\begin{array}{l}\text { Median duration of symptoms } \\
\text { before therapy, day }\end{array}$ & $7(4-12)$ & $6(3.5-11)$ & $9(4-12)$ & 0.214 \\
\hline \multicolumn{5}{|l|}{ Symptoms } \\
\hline Cough & $57(87.7)$ & $28(90.3)$ & $29(85.3)$ & 0.812 \\
\hline Sputum & $45(69.2)$ & $22(71.0)$ & $23(67.6)$ & 0.983 \\
\hline Fever & $43(66.2)$ & $23(74.2)$ & $20(58.8)$ & 0.296 \\
\hline Dyspnea & $40(61.5)$ & $24(77.4)$ & $16(47.1)$ & 0.024 \\
\hline Diarrhea & $22(33.8)$ & $11(35.5)$ & $11(32.4)$ & 0.997 \\
\hline Headache & $21(32.3)$ & $10(32.3)$ & $11(32.4)$ & 1.000 \\
\hline Sore throat & $18(27.7)$ & $8(25.8)$ & $10(29.4)$ & 0.963 \\
\hline Myalgia & $14(21.5)$ & $9(29.0)$ & $5(14.7)$ & 0.271 \\
\hline Chest pain & $9(13.8)$ & $4(12.9)$ & $5(14.7)$ & 1.000 \\
\hline Rhinorrhea & $8(12.3)$ & $5(16.1)$ & $3(8.8)$ & 0.605 \\
\hline \multicolumn{5}{|l|}{ Seven-category scale at baseline } \\
\hline $\begin{array}{l}\text { 3: Hospitalization, not requiring } \\
\text { supplemental oxygen }\end{array}$ & $39(60.0)$ & $12(38.7)$ & $27(79.4)$ & 0.003 \\
\hline $\begin{array}{l}\text { 4: Hospitalization, requiring } \\
\text { supplemental oxygen }\end{array}$ & $24(36.9)$ & $18(58.1)$ & $6(17.6)$ & \\
\hline $\begin{array}{l}\text { 5: Hospitalization, requiring HFNC } \\
\text { or noninvasive mechanical ventilation }\end{array}$ & $2(3.1)$ & $1(3.2)$ & $1(2.9)$ & \\
\hline \multicolumn{5}{|l|}{ Seven-category scale at day 7} \\
\hline $\begin{array}{l}\text { 3: Hospitalization, not requiring } \\
\text { supplemental oxygen }\end{array}$ & $32(49.2)$ & $8(25.8)$ & $24(70.6)$ & 0.001 \\
\hline $\begin{array}{l}\text { 4: Hospitalization, requiring } \\
\text { supplemental oxygen }\end{array}$ & $31(47.7)$ & $22(71.0)$ & $9(26.5)$ & \\
\hline $\begin{array}{l}\text { 5: Hospitalization, requiring HFNC } \\
\text { or noninvasive mechanical ventilation }\end{array}$ & $2(3.1)$ & $1(3.2)$ & $1(2.9)$ & \\
\hline \multicolumn{5}{|l|}{ Concurrent treatment } \\
\hline Antibiotic agent & $60(92.3)$ & $31(100)$ & $29(85.3)$ & 0.079 \\
\hline Glucocorticoid & $4(6.2)$ & $2(6.5)$ & $2(5.9)$ & 1.000 \\
\hline IV immunoglobulin & $3(4.6)$ & $3(9.7)$ & $\circ(0)$ & 0.206 \\
\hline \multicolumn{5}{|l|}{$\begin{array}{l}\text { Nonenhanced chest CT imaging at } \\
\text { baseline }\end{array}$} \\
\hline No active lung lesion & $10(15.4)$ & $2(6.5)$ & $8(23.5)$ & 0.078 \\
\hline $\begin{array}{l}\text { Unilateral multifocal GGOs and/or } \\
\text { consolidations }\end{array}$ & $7(10.8)$ & $2(6.5)$ & $5(14.7)$ & \\
\hline $\begin{array}{l}\text { Bilateral multifocal GGOs and/or } \\
\text { consolidations }\end{array}$ & $47(72.3)$ & $27(87.1)$ & $20(58.8)$ & \\
\hline \multicolumn{5}{|l|}{ Laboratory values at baseline } \\
\hline White blood cell count, $/ \mu \mathrm{L}$ & $5,200(4,500-6,400)$ & $6,100(4,700-7,250)$ & $5,050(4,100-5,800)$ & 0.037 \\
\hline
\end{tabular}


Table 1. Continued

\begin{tabular}{|c|c|c|c|c|}
\hline Characteristic & $\begin{array}{c}\text { Total } \\
(\mathrm{n}=65)\end{array}$ & $\begin{array}{l}\text { Lopinavir-ritonavir } \\
\qquad(\mathrm{n}=31)\end{array}$ & $\begin{array}{l}\text { Hydroxychloroquine } \\
(\mathrm{n}=34)\end{array}$ & $p$ value \\
\hline Lymphocyte count, $/ \mu \mathrm{L}$ & $1,108(804-1,436)$ & $927(738-1,119)$ & $1,284(1,086-1,596)$ & 0.001 \\
\hline C-reactive protein, $\mathrm{mg} / \mathrm{L}$ & $21.7(5.5-60.9)$ & $48.2(21.5-78.8)$ & $8.7(2.0-26.3)$ & $<0.001$ \\
\hline Aspartate aminotransferase, U/L & $25(20-38)$ & $28(22-29)$ & $24(16-32)$ & 0.008 \\
\hline Alanine aminotransferase, U/L & $19(14-32)$ & $24(15-54)$ & $17(11-25)$ & 0.015 \\
\hline Serum creatinine, mg/dL & $0.7(0.6-0.9)$ & $0.8(0.7-0.9)$ & $0.7(0.6-0.9)$ & 0.278 \\
\hline \multicolumn{5}{|l|}{ Coexisting conditions } \\
\hline Diabetes & $14(21.5)$ & $7(22.6)$ & $7(20.6)$ & 1.000 \\
\hline Hypertension & $12(18.5)$ & $8(25.8)$ & $4(11.8)$ & 0.255 \\
\hline Cardiovascular disease ${ }^{a}$ & $15(23.1)$ & $7(22.6)$ & $8(23.5)$ & 1.000 \\
\hline Chronic lung disease $^{\mathrm{b}}$ & $13(20.0)$ & $7(22.6)$ & $6(17 \cdot 6)$ & 0.852 \\
\hline Chronic kidney disease & $4(6.2)$ & $3(9.7)$ & $1(2.9)$ & 0.540 \\
\hline
\end{tabular}

Values are presented as mean $\pm \mathrm{SD}$, number (\%), or interquartile range.

HFNC, high-flow nasal cannula; IV, intravenous; CT, computed tomography; GGO, grand-glass opacity

${ }^{a}$ Cardiovascular disease included ischemic heart disease, valvular heart disease, heart failure, arrhythmia, and cerebrovascular accident.

${ }^{\mathrm{b}}$ Chronic lung disease included asthma, chronic obstructive lung disease, interstitial lung disease, tuberculosis-associated lung damage, and lung cancer.

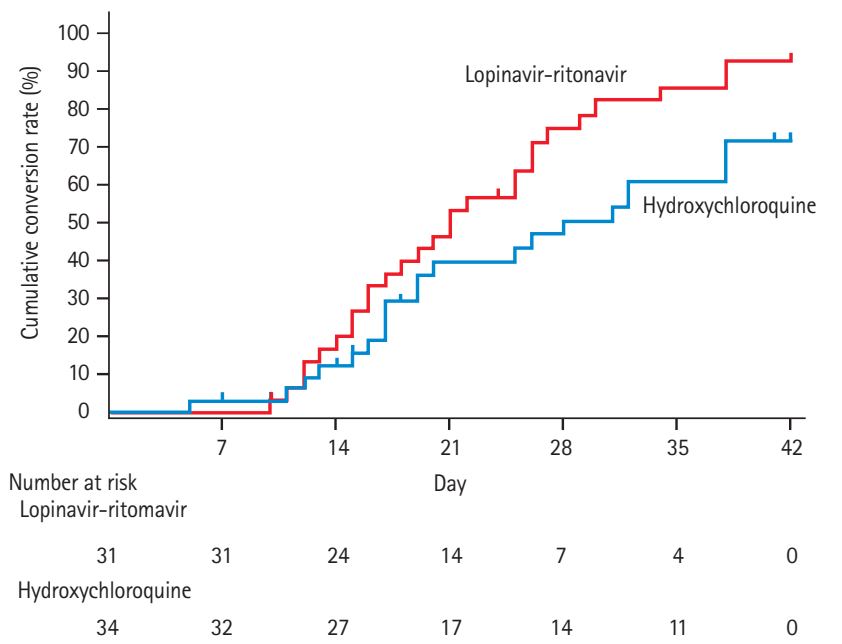

Figure 2. Kaplan-Meier curves showing time to negative conversion of viral RNA in nasopharyngeal and oropharyngeal specimens.

not significantly different between patients receiving lopinavir-ritonavir and those receiving hydroxychloroquine (median, 18 days vs. 21 days; log-rank, $p=0.216$ ) (Fig. 3). We investigated whether lopinavir-ritonavir treatment, as well as age, sex, duration of symptoms, ox- ygen support, concomitant therapy, chest CT findings, lymphopenia, elevated CRP, elevated AST or ALT, and coexisting conditions were associated with clinical improvement in the study population, but none of the variables were associated with clinical improvement (data not shown).

\section{Safety}

Adverse events tended to occur more frequently in the lopinavir-ritonavir group than in the hydroxychloroquine group, but this trend was not statistically significant(Table3). Lymphopenia and increased total bilirubin (of any grade of severity) were more frequently detected in patients treated with lopinavir-ritonavir. One serious adverse event (ARDS) occurred in one patient treated with lopinavir-ritonavir, and two serious adverse events (one event of ARDS and one event of shock) occurred in patients treated with hydroxychloroquine.

\section{DISCUSSION}

To our knowledge, this retrospective cohort study is 
Table 2. Cox proportional hazards regression investigating factors associated with negative conversion of viral RNA

\begin{tabular}{|c|c|c|c|c|}
\hline \multirow{2}{*}{ Variable } & \multicolumn{2}{|l|}{ Univariable } & \multicolumn{2}{|l|}{ Multivariable } \\
\hline & Unadjusted HR (95\% CI) & $p$ value & Adjusted HR (95\% CI) & $p$ value \\
\hline Age $\leq 65 \mathrm{yr}$ & $2.18(1.22-3.91)$ & 0.008 & $2.64(1.43-4.87)$ & 0.002 \\
\hline Male sex & $1.33\left(0.75^{-2.36)}\right.$ & 0.335 & & \\
\hline Use of lopinavir-ritonavir & $1.85(1.04-3.30)$ & 0.037 & $2.28(1.24-4.21)$ & 0.008 \\
\hline Duration of symptoms before therapy $<7$ days & $1.00(0.56-1.78)$ & 0.988 & & \\
\hline Oxygen support at baseline & $0.99(0.56-1.76)$ & 0.979 & & \\
\hline Antibiotic agent & $0.86(0.31-2.39)$ & 0.766 & & \\
\hline Glucocorticoid & $0.57(0.08-4.17)$ & 0.583 & & \\
\hline IV immunoglobulin & $3.00(0.71-12.71)$ & 0.135 & & \\
\hline $\begin{array}{l}\text { Bilateral multifocal GGOs and/or consolidations on } \\
\text { chest CT images }\end{array}$ & $1.15(0.61-2.18)$ & 0.667 & & \\
\hline White blood cell count $<5,500 / \mu \mathrm{L}$ & $1.03(0.58-1.83)$ & 0.928 & & \\
\hline Lymphocyte count $<1,000 / \mu \mathrm{L}$ & $1.58(0.89-2.81)$ & 0.120 & & \\
\hline C-reactive protein $>20 \mathrm{mg} / \mathrm{L}$ & $1.05(0.59-1.85)$ & 0.879 & & \\
\hline Aspartate aminotransferase $>35 \mathrm{U} / \mathrm{L}$ & $0.93(0.49-1.77)$ & 0.832 & & \\
\hline Alanine aminotransferase $>40 \mathrm{U} / \mathrm{L}$ & $0.80(0.37-1.71)$ & 0.565 & & \\
\hline Diabetes & $0.58(0.28-1.19)$ & 0.138 & & \\
\hline Hypertension & $1.45(0.74-2.86)$ & 0.284 & & \\
\hline Cardiovascular disease $\mathrm{a}^{\mathrm{a}}$ & $0.51(0.25-1.07)$ & 0.074 & & \\
\hline Chronic lung disease $^{\mathrm{b}}$ & $0.93(0.46-1.87)$ & 0.835 & & \\
\hline Chronic kidney disease & $1.19(0.43-3.30)$ & 0.746 & & \\
\hline
\end{tabular}

HR, hazard ratio; CI, confidence interval; IV, intravenous; GGO, ground-glass opacity; CT, computed tomography.

${ }^{a}$ Cardiovascular disease included ischemic heart disease, valvular heart disease, heart failure, arrhythmia, and cerebrovascular accident.

${ }^{\mathrm{b}}$ Chronic lung disease included asthma, chronic obstructive lung disease, interstitial lung disease, tuberculosis-associated lung damage, and lung cancer.

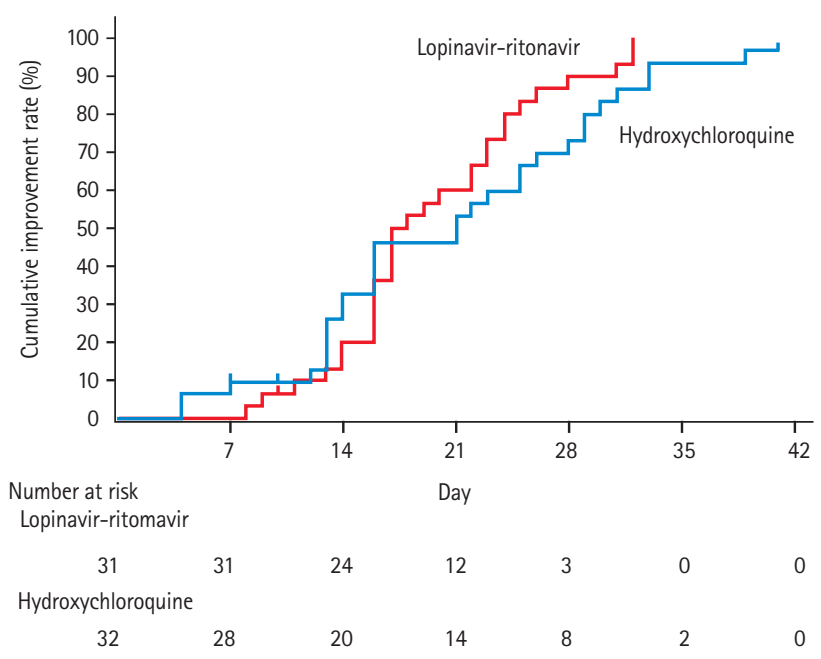

Figure 3. Kaplan-Meier curves showing time to clinical improvement. the first to compare the efficacy of lopinavir-ritonavir and hydroxychloroquine treatment in mild to moderate COVID-19 patients. Patients treated with lopinavir-ritonavir had a shorter time to negative conversion of viral RNA than patients treated with hydroxychloroquine, although time to clinical improvement did not differ between the two groups. Use of lopinavir-ritonavir and younger age was associated with negative conversion of viral RNA. In clinical practice, most patients with COVID-19 present with mild to moderate disease and their respiratory symptoms and vital signs improve with standard care. However, viral shedding persists for a longer period in some patients, which delays de-isolation and prolongs hospital stay. Because lopinavir-ritonavir-treated patients achieved more rapid viral clearance compared with hydroxychloroquine-treated patients, lopinavir-ritonavir 
Table 3. Summary of adverse events

\begin{tabular}{|c|c|c|c|c|}
\hline \multirow{2}{*}{ Variable } & \multicolumn{2}{|c|}{ Lopinavir-ritonavir } & \multicolumn{2}{|c|}{ Hydroxychloroquine } \\
\hline & Any grade & Grade 3 or 4 & Any grade & Grade 3 or 4 \\
\hline Any adverse event & $29(93.5)$ & $4(12.9)$ & $26(76.5)$ & $4(11.8)$ \\
\hline Neutropenia & $2(6.5)$ & o & $1(2.9)$ & $1(2.9)$ \\
\hline Lymphopenia & $14(45.2)^{\mathrm{a}}$ & $4(12.9)$ & $3(8.8)^{\mathrm{a}}$ & o \\
\hline Anemia & $16(51.6)$ & $1(3.2)$ & $15(44 \cdot 1)$ & $1(2.9)$ \\
\hline Thrombocytopenia & $3(9.7)$ & $\mathrm{O}$ & $4(11.8)$ & 0 \\
\hline Increased AST & $9(29.0)$ & $1(3.2)$ & $7(20.6)$ & $1(2.9)$ \\
\hline Increased ALT & $10(32.3)$ & $1(3.2)$ & $10(29.4)$ & o \\
\hline Increased total bilirubin & $8(25.8)^{b}$ & $1(3.2)$ & $1(2.9)^{b}$ & o \\
\hline Increased creatinine & $3(9.7)$ & $\mathrm{O}$ & $6(17.6)$ & 0 \\
\hline Nausea & $6(19.4)$ & 0 & $2(5 \cdot 9)$ & 0 \\
\hline Vomiting & $3(9.7)$ & o & $\circ(0)$ & $\mathrm{O}$ \\
\hline Diarrhea & $7(22.6)$ & 0 & $6(17.6)$ & $2(5 \cdot 9)$ \\
\hline Abdominal discomfort & $1(3.2)$ & o & $\mathrm{o}(\mathrm{o})$ & $\mathrm{O}$ \\
\hline Loss of appetite & $1(3.2)$ & 0 & $1(2.9)$ & 0 \\
\hline Skin rash & $1(3.2)$ & 0 & $\mathrm{o}(\mathrm{o})$ & $\mathrm{O}$ \\
\hline Dizziness & $1(3.2)$ & 0 & $1(2.9)$ & o \\
\hline Delirium & $5(16.1)$ & 0 & $2(5.9)$ & $\mathrm{O}$ \\
\hline Serious adverse event & $1(3.2)$ & $1(3.2)$ & $2(5 \cdot 9)$ & $2(5 \cdot 9)$ \\
\hline Respiratory failure & $1(3.2)$ & $1(3.2)$ & $1(2.9)$ & $1(2.9)$ \\
\hline Shock & 0 & 0 & $1(2.9)$ & $1(2.9)$ \\
\hline
\end{tabular}

Values are presented as number (\%).

AST, aspartate aminotransferase; ALT, alanine aminotransferase

${ }^{\mathrm{a}} \mathrm{p}<0.01$.

${ }^{\mathrm{b}} \mathrm{p}<0.05$.

might be the preferred drug in the treatment of mild to moderate COVID-19, especially due to the lack of specific antiviral agents currently available. Although some adverse events were more common in patients treated with lopinavir-ritonavir, the frequencies of grade 3 or 4 adverse events and serious adverse events were comparable between the two groups.

Unlike a previous randomized, controlled, open-label trial of lopinavir-ritonavir (which enrolled patients with severe COVID-19) [10], our study population consisted of mild to moderate COVID-19 cases and their treatment was initiated earlier in the disease process (median duration of symptoms before lopinavir-ritonavir treatment, 6 days vs. 13 days, respectively). These factors may have been associated with the superior results in lopinavir-ritonavir-treated patients in our study. Al- though our study did not demonstrate that early treatment was beneficial to achieve viral clearance compared with late treatment, previous data support the early use of lopinavir-ritonavir. In a clinical study of SARS-CoV-1 infection, treatment with a combination of lopinavir-ritonavir and ribavirin resulted in significantly less frequent occurrence of ARDS or death, as well as marked reduction of viral loads, compared with ribavirin treatment [5]. Notably, the benefit of lopinavir-ritonavir was evident only when it was used as an initial therapy, not as a rescue therapy [21]. Taken together, earlier use of lopinavir-ritonavir in a milder disease might result in better outcomes in the treatment of COVID-19, but further research is required to confirm these findings.

Lopinavir-ritonavir and hydroxychloroquine have different mechanisms of action to inhibit SARS-CoV-2. 
The SARS-CoV-2 genome encodes non-structural proteins (e.g., 3-chymotrypsin-like protease [3 $\left.\mathrm{CL}^{\mathrm{pro}}\right]$, papain-like protease, helicase, and RdRp) and structural proteins (e.g., spike glycoprotein, envelope protein, and membrane protein) [22]. The enzyme ${ }_{3} \mathrm{CL}^{\mathrm{pro}}$, one of the key enzymes in the viral life cycle, cleaves polyproteins translated from viral RNA and thus mediates viral replication $[23,24]$. Lopinavir-ritonavir is known to inhibit the action of ${ }_{3} \mathrm{CL}^{\text {pro }}$ thereby interfering with coronavirus replication [25]. Hydroxychloroquine may have multiple mechanisms of action. Normal acidic conditions of endosomes and lysosomes enable endocytosed coronavirus to be processed and released into the host cell cytoplasm [26]. Hydroxychloroquine increases the $\mathrm{pH}$ of endosomes and lysosomes, and disrupts protein degradation and release of the viral genome required for viral infection and replication [27]. Hydroxychloroquine can also block viral entry into the host cells by interfering with glycosylation of angiotensin converting enzyme 2 (ACE2) receptors [13]. SARS-CoV-2 spike proteins bind to ACE receptors on the cell membrane to enter the host cells [28]. However, it is not clear if these different mechanisms of action were associated with the superior results achieved with lopinavir-ritonavir treatment in our study.

Both lopinavir-ritonavir-treated patients and hydroxyhloroquine-treated patients achieved clinical improvement at a similar timepoint, but viral clearance was achieved earlier in lopinavir-ritonavir-treated patients. Currently, guidelines do not recommend a specific drug as first choice in the treatment of COVID-19. Infectious Diseases Society of America Guidelines recommend hydroxychloroquine or lopinavir-ritonavir to treat COVID-19, only in the context of a clinical trial [29]. Randomized, controlled trials of lopinavir-ritonavir and hydroxychloroquine are ongoing in the USA, Canada, Europe, China, and Korea. Results from these clinical trials could support the findings of our study.

Overall adverse events were comparable between the two drugs, except for lymphopenia and hyperbilirubinemia. Lymphopenia and hyperbilirubinemia were more frequently reported in patients treated with lopinavir-ritonavir. These adverse events were mild and transient. The higher incidence of lymphopenia and hyperbilirubinemia in the lopinavir-ritonavir group might be due to more severe cases included in this treatment group [2].

The present study is unique in that viral shedding was monitored serially for 6 weeks after the start of lopinavir-ritonavir or hydroxychloroquine treatment. Given that the median duration of viral shedding was 20 days and duration of viral shedding ranged from 8 to 37 days in survivors of COVID-19 [3], we designed this study to observe the patients for a sufficient period of time. Most previous clinical studies had observation periods for 5 to 7 days after initiation of treatment, and a few assessed patients through 28 days. Moreover, our study population is unique because more than $50 \%$ of hospitalized patients with COVID-19 were treated with only one of either lopinavir-ritonavir or hydroxychloroquine. This enabled the researchers to directly compare the efficacy of the two drugs.

The present study has some limitations. First, it was a retrospective and single-center design. Multi-center, randomized, controlled trials would be required to confirm our findings. Second, because this was a retrospective cohort study, the baseline characteristics of the patients were not perfectly balanced between those receiving lopinavir-ritonavir or hydroxychloroquine. Patients treated with lopinavir-ritonavir were more likely to have pneumonia confirmed on chest CT scans, and therefore required higher rates of oxygen support and more frequently complained of dyspnea. Despite the relatively more severe cases in the lopinavir-ritonavir group, the study outcomes suggest lopinavir-ritonavir may provide greater benefit than hydroxychloroquine.

In conclusion, we have compared virological and clinical responses to lopinavir-ritonavir or hydroxychloroquine treatment in mild to moderate COVID-19 cases. Patients receiving lopinavir-ritonavir had more rapid viral clearance than those receiving hydroxychloroquine, but there was no significant benefit in terms of clinical responses. Use of lopinavir-ritonavir and age were independent factors associated with viral clearance. Our findings need to be confirmed in randomized, controlled trials.

\section{KEY MESSAGE}

1. Use of lopinavir-ritonavir was associated with a better outcome in terms of viral clearance than use of hydroxychloroquine in patients with coronavirus disease 2019 (COVID-19). 
2. Clinical responses were comparable in lopinavir-ritonavir- and hydroxychloroquine-treated COVID-19 patients.

\section{Conflict of interest}

No potential conflict of interest relevant to this article was reported.

\section{Acknowledgments}

This study was supported by a grant from Research Institute of Medical Science, Catholic University of Daegu (2020) (received by Ji-Won Kim) and a research grant from Daegu Medical Association COVID-19 scientific committee (received by Hyo-Lim Hong). We thank all doctors, nurses, and healthcare professionals who participated in the care for patients with COVID-19 at Daegu Catholic University Medical Center. Also, we thank Dr. Jun Sik Yoon for giving statistical support and reviewing the manuscript.

\section{REFERENCES}

1. Chen N, Zhou M, Dong X, et al. Epidemiological and clinical characteristics of 99 cases of 2019 novel coronavirus pneumonia in Wuhan, China: a descriptive study. Lancet 2020;395:507-513.

2. Wang $\mathrm{D}, \mathrm{Hu} \mathrm{B}, \mathrm{Hu} \mathrm{C}$, et al. Clinical characteristics of 138 hospitalized patients with 2019 novel coronavirus-infected pneumonia in Wuhan, China. JAMA 2020;323:1061-1069.

3. Zhou F, Yu T, Du R, et al. Clinical course and risk factors for mortality of adult inpatients with COVID-19 in Wuhan, China: a retrospective cohort study. Lancet 2020;395:10541062.

4. World Health Organization. Coronavirus disease (COVID-19) pandemic [Internet]. Geneva (CH): WHO, 2020 [cited 2020 May 28]. Available from: https://www.who.int/emergencies/ diseases/novel-coronavirus-2019.

5. Chu CM, Cheng VC, Hung IF, et al. Role of lopinavir/ ritonavir in the treatment of SARS: initial virological and clinical findings. Thorax 2004;59:252-256.

6. Chen F, Chan $\mathrm{KH}$, Jiang Y, et al. In vitro susceptibility of 10 clinical isolates of SARS coronavirus to selected antiviral compounds. J Clin Virol 2004;31:69-75.

7. de Wilde AH, Jochmans D, Posthuma CC, et al. Screening of an FDA-approved compound library identifies four smallmolecule inhibitors of Middle East respiratory syndrome coronavirus replication in cell culture. Antimicrob Agents Chemother 2014;58:4875-4884.

8. Lim J, Jeon S, Shin HY, et al. Case of the index patient who caused tertiary transmission of COVID-19 infection in Korea: the application of lopinavir/ritonavir for the treatment of COVID-19 infected pneumonia monitored by quantitative RT-PCR. J Korean Med Sci 2020;35:e79.

9. Young BE, Ong SWX, Kalimuddin S, et al. Epidemiologic features and clinical course of patients infected with SARSCoV-2 in Singapore. JAMA 2020;323:1488-1494.

10. Cao B, Wang Y, Wen D, et al. A trial of lopinavir-ritonavir in adults hospitalized with severe COVID-19. N Engl J Med 2020;382:1787-1799.

11. Savarino A, Boelaert JR, Cassone A, Majori G, Cauda R. Effects of chloroquine on viral infections: an old drug against today's diseases? Lancet Infect Dis 2003;3:722-727.

12. Keyaerts E, Vijgen L, Maes P, Neyts J, Van Ranst M. In vitro inhibition of severe acute respiratory syndrome coronavirus by chloroquine. Biochem Biophys Res Commun 2004; 323:264-268.

13. Vincent MJ, Bergeron E, Benjannet $\mathrm{S}$, et al. Chloroquine is a potent inhibitor of SARS coronavirus infection and spread. Virol J 2005;2:69.

14. Biot C, Daher W, Chavain N, et al. Design and synthesis of hydroxyferroquine derivatives with antimalarial and antiviral activities. J Med Chem 2006;49:2845-2849.

15. Wang M, Cao R, Zhang L, et al. Remdesivir and chloroquine effectively inhibit the recently emerged novel coronavirus (2019-nCoV) in vitro. Cell Res 2020;30:269-271.

16. Yao X, Ye F, Zhang M, et al. In vitro antiviral activity and projection of optimized dosing design of hydroxychloroquine for the treatment of severe acute respiratory syndrome coronavirus 2 (SARS-CoV-2). Clin Infect Dis 2020;71:732-739.

17. Chen J, Liu D, Liu L, et al. A pilot study of hydroxychloroquine in treatment of patients with moderate COVID-19. Zhejiang Da Xue Xue Bao Yi Xue Ban 2020;49:215-219.

18. Gautret P, Lagier JC, Parola P, et al. Hydroxychloroquine and azithromycin as a treatment of COVID-19: results of an open-label non-randomized clinical trial. Int J Antimicrob Agents 2020;56:105949.

19. Gautret P, Lagier JC, Parola P, et al. Clinical and microbiological effect of a combination of hydroxychloroquine and azithromycin in 80 COVID-19 patients with at least a six-day follow up: a pilot observational study. Travel Med Infect Dis 
2020;34:101663.

20. Molina JM, Delaugerre C, Le Goff J, et al. No evidence of rapid antiviral clearance or clinical benefit with the combination of hydroxychloroquine and azithromycin in patients with severe COVID-19 infection. Med Mal Infect 2020;50:384.

21. Chan KS, Lai ST, Chu CM, et al. Treatment of severe acute respiratory syndrome with lopinavir/ritonavir: a multicentre retrospective matched cohort study. Hong Kong Med J 2003;9:399-406.

22. Li G, De Clercq E. Therapeutic options for the 2019 novel coronavirus (2019-nCoV). Nat Rev Drug Discov 2020;19:149150.

23. Anand K, Ziebuhr J, Wadhwani P, Mesters JR, Hilgenfeld R. Coronavirus main proteinase (3CLpro) structure: basis for design of anti-SARS drugs. Science 2003;300:1763-1767.

24. Zhang L, Lin D, Sun X, et al. Crystal structure of SARS-CoV-2 main protease provides a basis for design of improved $\alpha$-ketoamide inhibitors. Science 2020;368:409-412.

25. Rajnarayanan RV, Dakshanamurthy S, Pattabiraman N.

"Teaching old drugs to kill new bugs": structure-based discovery of anti-SARS drugs. Biochem Biophys Res Commun 2004;321:370-378.

26. Wang H, Yang P, Liu K, et al. SARS coronavirus entry into host cells through a novel clathrin- and caveolaeindependent endocytic pathway. Cell Res 2008;18:290-301.

27. Devaux CA, Rolain JM, Colson P, Raoult D. New insights on the antiviral effects of chloroquine against coronavirus: what to expect for COVID-19? Int J Antimicrob Agents 2020;55:105938.

28. Hoffmann M, Kleine-Weber H, Schroeder S, et al. SARSCoV-2 cell entry depends on ACE2 and TMPRSS2 and is blocked by a clinically proven protease inhibitor. Cell 2020;181:271-280.

29. Bhimraj A, Morgan RL, Shumaker AH, et al. Infectious Diseases Society of America guidelines on the treatment and management of patients with COVID-19 [Internet]. Arlington (VA): Infectious Diseases Society of America, 2020 [cited 2020 May 28]. Available from: https://www.idsociety. org/practice-guideline/covid-19-guideline-treatment-andmanagement/. 